\title{
Internet use, in- and exclusion in decision-making processes within political parties Gefion Thuermer ${ }^{1}$ Silke Roth ${ }^{1}$ Markus Luczak-Rösch ${ }^{2}$ Kieron O'Hara ${ }^{2}$ ${ }^{1}$ Social Sciences \\ University of Southampton \\ ${ }^{2}$ Electronics and Computer Science \\ Southampton, UK \\ \{gefion.thuermer, silke.roth, m.luczak-rosch, K.M.O'Hara\} @soton.ac.uk
}

\begin{abstract}
This paper investigates the effect of internet-use on democratic decision-making processes within political parties. Through two case studies of the Green Party and the Pirate Party Germany, the influence of internet-use on these processes and their inclusiveness are shown. We argue that how the internet is used in democratic processes impacts on participation and inclusion.

How internet technology interacts with decision making processes within parties depends on the existing party structure and culture. Thus, in order to achieve meaningful and inclusive participation, the institutional framework and the influence it has must be considered in process and tool design. Whereas the affordances of specific online tools have been evaluated, the institutional context in which they are embedded have so far been widely ignored. We offer a structure for analysis of these foundations.
\end{abstract}

\section{CSS Concepts}

$\cdot$ Human-centered computing $\rightarrow$ Empirical studies in HCI

•Human-centered computing $\rightarrow$ Empirical studies in collaborative and social computing

\section{Keywords}

Decision-making; e-democracy; political parties; process design; inclusion

\section{INTRODUCTION}

Political parties are a fundamental building block of democratic societies. Citizens form parties to influence the policy-making process, which in turn ultimately shapes the laws that govern society. If - following Hyland [20] - democracy is understood as giving equal opportunities of participation to every citizen, then parties are one route through which this participation is enacted. This means that decisions within political parties should be made democratically as well. Parties have structured and documented decision-making processes, which include steps such as the development of ideas, proposition of motions or candidates, discussion and deliberation, and concluding votes. Some of these happen in a formal setting, governed by regulations, others are very informal; some of them take place on the internet, others offline. Due to the constant requirement for democratic decisions in politics, parties are an excellent organisational type to study democratic decision-making. According to Hyland, a decisionmaking process "must have a rule that determines the decision to

\footnotetext{
Permission to make digital or hard copies of all or part of this work for personal or classroom use is granted without fee provided that copies are not made or distributed for profit or commercial advantage and that copies bear this notice and the full citation on the first page. Copyrights for components of this work owned by others than the author(s) must be honored. Abstracting with credit is permitted. To copy otherwise, or republish, to post on servers or to redistribute to lists, requires prior specific permission and/or a fee. Request permissions from Permissions@acm.org. WebSci '16, May 22 - 25, 2016, Hannover, Germany

Copyright is held by the owner/author(s). Publication rights licensed to ACM. ACM 978-1-4503-4208-7/16/05...\$15.00
}

DOI: http://dx.doi.org/10.1145/2908131.2908149 be implemented as a function of the decisions made (...) by each participant" [20:41]. In consequence, decisions need to be made by all party-members, so that participation is indeed equal.

Many studies have discussed how the internet is used in parties or in decision-making processes, and how political communication has changed through the internet. For example, Kerr and Waddington [23] described the implementation of online branches in a trade union, Jackson and Lilleker [22] investigated the effect social media has on political communication, and Gibson and Cantijoch [10] compared online and offline participation. However, to date there has been little research specifically on the role the internet plays and how it influences the decision-making processes in parties themselves.

The two parties considered in this paper are the Green Party and Pirate Party Germany. Both parties were chosen due to their age, ideology, and advocacy for grassroots-democracy, all of which make them particularly suitable for this comparison. The Green Party was founded by environmental, peace, and women's movements activists, just before the rise of the web in 1980 . Although their ideals shifted during more than 30 years of political work, some of the grassroots-ideals still remain. The party wants to benefit from online technology, but also make sure that no-one who may not use the internet is left behind. The Pirate Party was founded as part of a global movement against the criminalisation of online culture in 2006, and strives for participation of all members. It developed quite literally through the internet, and publishes virtually all documents relevant for decisions online. The party believes that through new technology like the internet, democratic processes should change - and consequently uses a vast array of online tools through which members can participate.

Both parties went through similar organisational developments, but at different times. Their decision-making processes are founded on grassroots and dense participation, but differ strongly in their implementation: The Green Party uses representation for most decisions beyond the local level, and has strict regulations for participation such as women's quotas, whereas the Pirate Party allows participation for everyone in most processes, and direct voting for all members in all decisions relevant to their locale, but no representation at all. Both parties follow agendas on the left political spectrum, but are very different in their processes and use of technology, they offer a good comparison as organisations. Many comparisons have been drawn between them in the past [39], however none of these have looked at the parties' decision-making processes, and the role of the internet in these processes.

Different internet services (email, VoIP, web) are used within parties. Since we cannot discuss the implications of different protocols in this paper, and the distinction made in practice is between 'online' and 'offline' while the specific type of 'online' is of little meaning to the users, we will focus on the internet as a whole. 
The internet, especially the web, offers the opportunity for everyone to easily contribute content and engage in discussions. This function is often perceived as a game changer, which influences how information is distributed and retrieved. We argue that this assessment passes over inequalities that arise through the technology. Both parties try to achieve similar goals, but they use different forms of participation, both on- and offline. The differences identified through the case studies, such as representative democracy versus direct participation, and 'collective or connective action' [3], are linked to their ideological beliefs and institutional foundations. Insights gained through their comparison can help guide the design of future online participation platforms, and studies about these platforms, but also have wider implications for studies of innovative democratic concepts.

\section{BACKGROUND}

Hyland describes democracy as the form of political rule where "all those significantly affected by the decisions have equal and effective rights of participation at all levels of decision-making" [20:2]. In practice, this requires consideration of the 'who' and 'how' of decision-making. Party membership is defined through laws and statutes, which creates a bounded group for the former, whereas the latter will be investigated through the remainder of this paper. The rules around decision-making in political parties more and more often involve online elements. The effect of these elements on the democracy of the decisions will be discussed in the following.

\subsection{Democratic Decisions}

Group decision-making can be conducted in various ways, all of which share advantages, such as

\begin{abstract}
"More knowledge and expertise is available to solve the problem; a greater number of alternatives are examined; the final decision is better understood and accepted by all group members; and there is more commitment among all group members to make the final decision work." [27, in 35]
\end{abstract}

However, group decision-making is also prone to some disadvantages, including social pressure (for individuals to decide in a certain way), elite domination (of powerful individuals over the group), and time delays (the larger the group the more time is required for all to have their say and agree). Organisations find different ways to use the advantages and work around the disadvantages, and political parties specifically try to overcome some of these challenges through the use of online technology.

Where organisations used to organise hierarchically, they can now be observed to apply network structures instead. In the context of social movements, Bennett and Segerberg [3] describe this as the difference between the logic of collective and connective action. They observe the emergence of connective action, which is supported by, but not solely achieved through, internet technology. Connective action networks consist of individuals who share goals, and function with little to no coordination, through information exchange and the sharing of online content. Participation is the core of the network, not a means to an end, and formal organisations are neither required, nor necessary. In organisations of collective action, activity is structured centrally. The base problem is to recruit people who contribute to a common cause, for which well organised and funded organisations are required.

We suggest that the logics of collective and connective action could be applied to parties, or their members, and to the way in which parties organise: On the one hand they are required to have a formal structure, which is very much on the collective action side of the spectrum, while they may also want participation at the centre of their political work, and thus encourage their members to act as connective action networks. This is likely more so if parties stress bottom-up members' participation over top-down decisions.

Democratic decisions require equality and participation of all affected, either directly or through representatives. Inequalities occur when specific groups or minorities are not represented in, or systematically excluded from, the decision-making process. This can happen through personal, social and economic circumstances of participants, or the practical implementation of processes. They can also be inherent in organisational processes. One example for this is the systematic discrimination of women through supposedly gender-neutral standards organisations have developed based on men, through underlying assumptions that are hardly questioned, as described by Acker [1]. Moreover, voting behaviour correlates with education and income, and reflects gender-segregated labour markets [25]. Parties who want to make democratic decisions need to be aware of these issues in order to address and mitigate them.

Processes achieve equal participation in different ways. Voting, the typical decision-making process in democracies, merely requires standing opinions that can be voiced and counted [41]. A vote may be preceded by discussion, which helps individuals to form an opinion based on which they then cast their vote. Consensus is neither achieved, nor is it necessary. In theory, equal participation is given so long as everyone has a vote. Whether this is truly the case in practice depends on the actual implementation of the process by which binding decisions are made.

In deliberative processes, participants exchange opinions and find consensus in open discourse. Deliberation requires the exchange of arguments and opinions, and scrutiny of different points of views. Genuine openness to changes of opinion is paramount, since the goal is to agree on a consensus acceptable to all, and not to convince another party of a specific point of view.

In both consensus and deliberative decision-making, the basis for equality is the equal opportunity to be heard. This was found to be problematic in the Occupy movement [36], which used consensus decision-making at their assemblies. Despite the goal of promoting equality, the process in practice leads to exclusion: the only way for members to participate in decisions is to attend assemblies. Time and location of these assemblies put members who have other commitments at a disadvantage. Among those who do attend assemblies, the less experienced members can be discouraged from active participation. Moreover, participating in decision-making as well as in working groups is complicated, as schedules of assemblies and group-work overlap. Thus members have to choose whether they want to actively work for the movement in these groups, or attend assemblies to participate in its decision-making [36]. This has been recognized by Occupy and other actors who engage in consensus-decision making, and the 'progressive stack' (in which members of underrepresented groups (women, ethnic minorities) are prioritised in speakers' lists) are employed to assure that these groups are included [34].

Such social movements often claim to be leader-full rather than leader-less, because many members are involved in decision making processes [8]. It is assumed that this prevents a rule through elites and improve equal participation. This is not necessarily the case however, because instead of staying 'structureless', informal structures can evolve. This can in turn lead to informal elites who influence decisions, but have no obligation to act in the interest of 
the group. Without formal structures, these informal elites cannot be held accountable [38]. Furthermore, the absence of representatives that can speak for the organisation and lead discussions can be a disadvantage in organisation applying consensus decision-making.

\subsection{Use of Technology}

Both voting and deliberation are supposedly simplified through the internet. However, participating online requires technical capital, which is not equally distributed, because it is linked to similarly unequally distributed social and economic capital. Already disadvantaged groups do not have large amounts of these forms of capital, so they stay disadvantaged when they go online [43]. The internet, though it may increase power for example for global media corporations or talented 'geeks', also reproduces existing inequalities for the illiterate, the poor, and otherwise disadvantaged groups.[15]

Provision of hardware, internet access and training can help, but not solve the problem of unequal usage on their own, because users can only apply skills usefully in a context that they are familiar with. Therefore, the use of internet and communication technology (ICT) needs to be grounded in and formed through everyday practice [14]. Internet use can increase political participation, as Ward et al. have found in the UK Liberal Democrats, albeit only if a base interest already exists [40].

In addition to differences in access, those who do have access to the internet also derive unequal benefits from its use $[16,18]$. Each group, online or offline, uses it in their own particular context: "different social institutions have different objectives, concerns and modus operandi, and hence impose different requirements on their IT infrastructure" [7:1]. Not only in Germany, the internet is primarily used by young, male and well-educated individuals, who are thus over-represented in online decision-making processes [26]. Gibson and Cantijoch found that most of the political participation that happens online are offline activities that have been transferred online, such as reading news [10].

In 1987, Held described how citizens should have more influence on issues that concern them locally, in a form of participatory democracy. ICT was seen to play a key role in this participation, especially for disadvantaged groups [19]. It would be an opportunity as well as a threat, and "could lead either to popular sovereignty or to populist manipulation”[29:56], depending on who uses it more effectively.

If online participation is to fulfil the democratic requirement of equal participation, all participants must be able to fully participate in decision-making processes. They need access to, and skills in the use of, the technology. If party members do not have access to all of these, online processes can create inequality.

\subsubsection{The internet in organisations}

Access is not only required for members to the internet, but also the other way around: the organisation must be able to reach all their members online, if online processes are to be inclusive. A study of the introduction of virtual branches in UNISON showed that a transition to online processes is surprisingly complicated [23]. Although nowadays an email address is typically collected along with address information, the union was founded before the internet, and thus did not have this data for all their members. The virtual branches increased participation, especially for members that had already been active offline, who then adopted the additional online service. Participation increased gradually beyond this group, especially for women, possibly pointing to increased use in the long term. However, the higher the diversity of members was, the higher was the workload required to maintain the virtual branches, and the workforce that had previously handled the offline processes was insufficient for the same task online.

Online participation is possible, but it does not come for free, and not on its own. Institutional processes are generally stable, they do not change through new technologies by themselves. They may change when new technology becomes available, but only through the integration of new tools into existing processes, which can either happen consciously intended, or unanticipated [2]. As Gibson et al pointed out: "Context matters", and the use organisations can make of internet technology will be limited by their size, age and function [11].

Discourse on online platforms also causes other concerns: Online platforms tend to be homogenous, as individuals with similar opinions find each other in groups where they mutually reinforce existing opinions rather than challenging them [41]. Online forums primarily facilitate monologues and self-expression, instead of dialogue and exchange of ideas [42]. Similarities with tendencies of social network platforms are evident: homogeneity on Facebook groups, and reinforcement of existing opinions not only through social selection of friends, but also through algorithms of the platform itself [5]. Networks that exist offline are replicated online, and individuals who participate online were typically already active offline, so no new audience is reached simply by going online [33].

Rather than opening the political sphere up to new groups, people who are already politically involved use the internet to improve on that work. Platform design determines what can be achieved, and is therefore a policy decision in itself [13, in 41]. Especially in parties, where members would be expected to have similar views already, all of this may be the case - and should be, according to David Weinberger [21], who argues that a 'base agreement' is necessary for conversations. Similarly, O'Hara and Stevens [32] point out that these 'echo chambers' are neither necessarily harmful, nor unique to the internet.

\subsubsection{Online decision-making}

Decision-making by anonymous citizens may lead to morally inacceptable decisions, because this group would not be accountable to anyone. The simple aggregation of opinions cannot replace the checks and balances of a representative democracy system, because parties as intermediaries are important. They "do not simply transmit information; they actively process it, especially by synthesizing political opinions and interests into ideologically coherent platforms" [2:312]. Replacing representative democracy with direct democracy through online platforms would neglect this role of parties and thereby weaken the democratic process.

The main barriers for direct participation are time and space limitations for face-to-face meetings, lack of (perceived) expertise, and time limitations to fill knowledge gaps and vote on all decisions. Millers 'program for direct and proxy voting in the legislative process' is an attempt to overcome all three of these barriers. As a hypothetic prototype for Liquid Democracy systems, it suggests to "allow citizens to vote directly or delegate proxy to someone else" [28:108, italics in original]. What Miller imagined to be modern computer technology in 1969 would allow to tally votes from delegates as well as individual voters on any matter.

Conceptually, Liquid Democracy allows the 'fluid' delegation of votes to 'proxies', who then vote on behalf of the constituent for specified areas or decisions. But it is prone to the same criticism as direct decisions: Although delegates may be held accountable by their constituents, participants who vote directly rather than 
delegating are not accountable to anyone. Instead of coherently grouped opinions, and the potential to negotiate consensus between groups, without representative elements, decisions would be made purely based on majorities and may thus lead to unethical results [9].

One attempt to implement Liquid Democracy is the software Liquid Feedback (LQFB), an online-voting tool that is popular within the Pirate Party movement. It allows to support motions, make alteration suggestions, and propose alternatives. Users can either vote on issues themselves, or delegate their vote for an issue, area, or the entire platform to another user. LQFB has been analysed in many contexts, however most research focuses on the software alone, without considering its wider position as part of a decision-making process [6, 24].

The biggest technical problem in Liquid Democracy systems is the requirement to allow secret votes, while at the same time giving users who delegate to others the possibility to hold their delegates to account, which requires their votes to be non-secret. Google Votes might be a solution for this problem. It was developed on the internal part of the Google+ social network, and lead to a 'Golden Rule of Liquid Democracy': "If I give you my vote, I can see what you do with it"[17:4]. This is based on the requirement that delegations must be explicitly accepted, which is not the case in LQFB.

Another option for deliberative online decision-making is the platform 'Deliberatorium', which uses argument mapping to structure discussion within groups. This allows more efficient deliberation at a larger scale opposed to what is possible in online forums [37]. The platform proved less time consuming for organisation as well as participants, since structure, filtering and elimination of redundancy - typical forum drawbacks - were done throughout the process. This improved the discussion and its result, and also improved the experience for users. Deliberation tools may well be the next step towards scalable online decision-making, although their scale is still limited.

\section{METHODOLOGY}

The case studies comprise the analysis of statutes and regulations, and in-depth interviews with members of both parties. The formal rules that govern both processes were drawn from the documents and formed the basis of the interview guide.

\subsection{Sampling}

The selection of interview partners was informed by the party structure. Selection criteria were be based on 'hot spots' in the party and process framework, such as members that were involved in committees or held positions which oversee processes. All interview partners have overseen, managed, participated, or otherwise been involved in the decision-making processes. Where possible, participants were approached through existing personal contacts. In addition, the party head offices were contacted to identify and reach out to suitable individuals.

Three interviews were conducted with actors from the Green Party, five interviews with actors from the Pirate Party. The number of interviews was limited by time constraints for the completion of the study, as well as by access to the relevant individuals. The latter is especially true for the Green Party, where fewer personal contacts existed. Despite the smaller number of interviews, the data gathered from the key stakeholders is sufficient to analyse the processes.

\subsection{Semi-Structured Interviews}

Qualitative interviews were considered the most appropriate approach to gain insight into the formal and informal elements of the decision-making processes, since only involved party members would know the latter, and the study required depth which could not have been gained through other means. All interviews were conducted via Skype, and lasted between one and two hours.

The interviews covered all aspects of the formal process. Open questions allowed to focus on areas that were important for the interviewees, or where they had specialist knowledge. Interview questions revolved around the decision-making processes for policy decisions and board elections. Participants were asked to describe what typically happens within and beyond the framework set by laws and statues, the role of the internet in the process, and its perceived strengths and weaknesses. The aim was to find out how the rules were applied and possibly modified in real-life situations, which additional elements of the process exist, what role the internet plays, and which inequalities are addressed. All participants were interviewed in German, and quotes in the following have been translated into English.

\subsection{Positionality}

The first author of this paper was a member of the Pirate Party for several years, amongst other activities also filling roles in the executive board. Through the active work in the party, the author already had extensive insight into the party processes, which played a role in the specific design of the study. Familiarity with several individuals made access into the Pirate Party easier - a benefit that did not exist to the same extend in the Green Party. Existence of this personal network was not perceived as having a negative influence on the interviews themselves, in either party. The familiarity with one parties' processes is why in-depth interviews were considered to be the best approach to this study. Not only did they allow to gain similar insight in both parties, but also gave us a foundation to base the analysis on others' rather than our own experience.

\subsection{Analysis}

All interviews were coded for six criteria:
1. Actions (What happens?)
2. Places (Where does it happen?)
3. Processes (How does it happen?)
4. Use of the internet (How is it used?)
5. Factors of consideration (Why does it happen this way?)
6. Inequalities (What is inclusive / exclusive?)

Since responses were based on the experience of interviewees at multiple positions within the process, codes 1-4 were mainly used to guide the development of a clearer picture of the entire process. Codes 5 and 6 were used to identify underlying themes.

Through the comparison of the decision-making processes of both parties, the following questions were to be answered:

- How does the use of the internet in decision-making processes differ between the parties?

- What effects do the different processes have regarding inclusion / exclusion?

\section{RESULTS}

Both parties see different forms of inequalities, and address them through different means, while claiming to exercise grass-roots democracy. The Green Party is very conscious of inequalities, and attempts to either reduce or at least not increase them. The Pirate 
Party aims to achieve equality by creating a uniform starting position for all members. While the Pirate Party intends to give as many members as possible the opportunity to be directly involved, through a variety of online tools, the Green Party tries to give every member an equal opportunity to participate, and thus limits the participation opportunities to the lowest common denominator among all members, which involves far less online technology and more traditional participation techniques.

\subsection{Democratic Decisions}

\subsubsection{Participation}

In the Green Party, the online distribution of motions is seen as one of the main administrative advantages. The party specifically created a tool, known as the Antragstool (motion tool) which serves as a platform for motion access and submission. It was initially created for work on policies, and includes a discussion process, through which motions could be collaboratively created. However the discussion function is deliberately not used, because it is considered impractical. Through this tool the decision-making processes became more transparent as now all members can access all motions for assemblies, and see what happens to the motions they submitted, both of which was previously impossible. This only benefits members who use the internet, but is still an improvement to what would have been possible before, when members could only access motions through paper files in their local branch office. However, according to the federal office, the number of motions that are proposed for each conference did not change since they are published and submitted online. Although the online tools have not achieved equality in this case, they reduced inequality and intensified, if not increased opportunities for participation.

In the Pirate Party, motions are developed, submitted, and discussed primarily online, through party owned or mediated platforms such as etherpads for collaborative writing, Mumble (a VoIP software) for voice conferences, email and mailing lists for group communication, a wiki to publish and archive information, survey tools for polls, and Liquid Feedback to refine motions. The party is not formally involved in the development or discussion of the motions, it just provides tools that everyone can use, and members - including board members in their 'rank-and-file member' role - organise themselves decentrally. Members can submit motions offline if they wish, but there is no offline way available to access them, let alone an offline way to find out how they could do this. All processes are so entirely internet-based, that pure offline participation may well be impossible.

\subsubsection{Representation}

In the Green Party, all members are represented in national decisions through their local representative. These are elected at regional assemblies, which gives the regions weight both in regards to party conferences and as a platform for discussion of motions. The local branch is the way for members to be represented and hold their representatives to account. Because delegates attend assemblies as official representatives, their travel costs are reimbursed.

The Pirate Party does not use a representation system for their assemblies because this would be considered incompatible with the ideal of grassroots democracy. Instead, all members can attend general assemblies and vote upon all issues themselves. Whether members do attend depends on their available time and money for travel, as the party does not reimburse these costs for attending rank-and-file members. The potential exclusion through travel and accommodation costs is frequently discussed, and while attempts are made to address it, for example through low-cost transport solutions or reduced rates at local hotels or campsites, a partymediated solution does not exist. If members cannot attend, they cannot participate.

Whether delegations, especially chain delegations (members delegating to members who delegate to other members) in online systems such as LQFB are acceptable, has been a tremendous discussion within the Pirate Party which has not yet been finally concluded. The party uses the internet to communicate across federal states; regional branches are therefore hardly relevant for decisions at the national level.

\subsubsection{Time}

The Green Party interviewees perceived significant differences between full-time politicians and volunteer members, mostly due to a difference in resources and time: full-time politicians can have their staff brief them on important motions. This resource is not available to volunteer members, who would have to go through all motions on their own, which is impossible due to their number (for the last assembly roughly 2,600), as a member of staff pointed out:
"About $60 \%$ of the participants of delegate assemblies are volunteer members. They don't sit in front of their computer every week to look at the preparations of the conference. They have a normal job, family, and might look at motions two weeks before."

This problem can only be partially solved. It is addressed through a discussion organised by the motion committee in advance of conferences, which aims to reduce the number of motions that is to be voted on. However, this in turn leads to lack of clarity, as change motions often overlap, which makes it complicated for members to stay aware of what they are deciding about, as a member of the party leadership 'described:
"Many change motions relate to a single line. If you accept the first motion, the line changes. But then there's another motion that changes the same line, and makes a completely different change. So you try to combine them somehow. (...) And then we have this 1600 times. This is really complicated to process, so that it is still transparent and democratic. (...) When people are not informed and vote on motions without really knowing what it is they are deciding about, because they cannot retrace it, it is doubtful whether this is still in accord with our democratic ideals."

This problem also exists in the Pirate Party, but since the party has full-time politicians only in four state parliaments, the difference to members is less problematic at the national level. The party can also only decide about a limited number of all submitted motions at each assembly (19\% of submitted motions were discussed at the most recent one). Rather than limiting their number, the party has developed an online process to rank the motions in advance of the assembly, by which means it then determines the assemblies' agenda. However the agenda can be changed through a vote by the assembly, which makes the motions that are going to be discussed unpredictable. This means that attendees would still need to prepare for all motions to be discussed. Whereas in the Green Party only the delegates need to prepare for the assembly, in the Pirate Party this applies to all members who wish to participate, which likely takes more factual time from other tasks. However, the assembly attendees have found ways to work around this, such as factual peer 
delegations: Often a handful of members will act as opinion leaders whose example other members follow during open votes.

\subsubsection{Gender}

For the Green Party, with roots in the women's movement, inequalities based on gender are seen and addressed throughout the decision-making process. This is achieved mainly through genderbased speakers' lists, who are called in turns, and a 50\% quota system for women across all elections. These gender-balancing practices are engrained and all interviewees seemed to see them as self-evident.

In the Pirate Party, gender is not addressed at all. Interviewees spoke about the fairness of the process, for example a member of the PR team stated:

\section{"The advantages of grass-roots democracy cannot be valued high enough (...) One is not just a paying member, but truly participates.”}

None of the five (and all male) interviewees even mentioned gender differences. At general assemblies, discussions are structured by opinion rather than gender: speakers are called alternately based on whether they want to argue for or against a motion. The Pirate Party does not collect data on gender but an independent study estimates that approximately $8.5 \%$ of the membership in 2011 identified as women [30]. Their lack of women in elected positions is striking, compared to the $50 \%$ quote of the Green Party. The Pirate Party Berlin just elected 32 candidates including 7 women for the next election [4]; the executive board has nine members including two women [31]. By way of comparison, the Green Party currently has the highest proportion of women in the larger German parties with $38.5 \%$ [31]. A women's quota has been discussed and refused by a Pirate Party assembly. There is no perception that women would have different or additional requirement for equal participation. Because women have the same opportunities in the process as men, their requirement for participation is perceived as satisfied.

\subsection{Use of technology}

\subsubsection{Formality}

In the Green Party technology and the internet are formal parts of processes and included in party regulations, such as the requirement for motions to be published online. The internet is used to amplify administrative processes, save costs, and increase participation. Everything happens offline by default. If anything is done online, it is a conscious decision, aiming to make processes faster and cheaper, and content more accessible for better participation.

Although technology and the internet are used extensively by the Pirate Party, their use is far less formalised. The internet is hardly even mentioned in the regulations. Purely based on party regulations, every process could be performed offline. This is not the case in practice however, as the internet is what makes these process and their scale possible in the first place. It is natural for the party to do everything online. A few exceptions are deliberately kept offline, such as assemblies for decisions, or elections being held on paper rather than digital.

The tools that are used for formal processes, such as the wiki to submit motions, are used only by custom: they are not even mentioned in the statutes or procedural rules. In board elections, candidates apply online, are introduced and questioned online. The party allows every member to stand for these elections, typically leading to a large number of candidates. The online preparation is therefore necessary, because otherwise every candidate would need to have extensive introduction time at the assembly, which would demand too much time. Overall, the use of offline techniques seems to be largely due to legal requirements (such as time for candidates to introduce themselves) or insufficiencies in technology (such as secure online voting systems).

\subsubsection{Online access}

Internet access of members is the main concern for the Green Party, as visible in the non-use of online discussions: It would be impossible to keep all members - including those that still want (and have a right to receive) all documents on paper - informed about the latest status of the motions, let alone the discussion. If not all members would be able to participate, in a process that is governed by the party, the party would generate inequality. The process would be very easy to do online, but cannot be made compatible to the offline world. To treat all members equally, online processes are not used at all - the smallest common denominator are the well-worn offline processes. As a member of the party leadership stated:

\section{"We need to transpose decision procedures to the web, and to understand who participates. What if it is only $1 \%$ of the member base, and so on? (...) Who would be excluded from this process? What about people who do not have a computer? It may not be many, but they do have different requirements."}

The Pirate Party considers all online processes to be accessible, and deliberately offers a variety of online platforms for participation. All of these enable the members to communicate and collaborate, draw up new motions, propose them, announce applications for elections, and campaign amongst the membership. As stated by a member of the team that organises general assemblies:
"The claim that there are members of the Pirate Party who are excluded from participation because they do not have internet access or a computer is coming up frequently. (...) despite searching for them, I have never seen this 'Offline-Pirate'."

The most striking difference between the parties' use of the internet are their reasons for this use: it is participation and equality for both of them. But while the Pirate Party uses the internet to achieve participation and equality, the Green Party does not use it for the same reason. While the Green Party avoids the use of online discussion platforms, as it would exclude members who do not have access to the internet, the Pirate Party employs them so that everyone can participate. This is based on a difference in fundamental assumptions of both parties. Where the Green Party assumes that not all members have access to the internet, the Pirate Party assumes that all their members do. However, the differences between the two parties are actually relatively small, $82 \%$ of the Green Party members and $92 \%$ of the Pirate Party members can be reached via email according to data provided by party administration.

This means that $8 \%$ of the members of the Pirate Party cannot be contacted by email. This does not mean that these members do not participate at all. Invitations to assemblies are sent by post for those members that cannot be reached online, and access to most systems does not require validation through the party, let alone membership. But the party cannot involve these $8 \%$ of their members in the two online process elements that are party-governed: official polls and access to Liquid Feedback. 


\subsubsection{Communication and Consensus}

The Green Parties' own online social network Wurzelwerk is used in different ways across the federal branches but not for discussions about the party conference. Discussions instead happen informally on privately owned sites such as Facebook, and on a face-to-face consensus-meeting the night before party conferences.

The Pirate Party does not support any formal face-to-face (or indeed any formal) meetings for consensus-finding. These are instead arranged by members themselves, through infrastructure provided by the party. Instead of proposing alternative motions, they are refined before they are submitted, and made capable of consensus through Liquid Feedback, where controversial elements can be spotted and resolved. However this can create consensus only among participating members on that platform - there is no data to confirm the overlap between the participants of Liquid Feedback and general assemblies.

Both parties' members use online social networks to discuss candidates and motions, but these discussions happen only through informal groups of members. Interviewees from the Pirate Party speak about online-tools as 'natural, taken for granted, totally normal'. Often the variety of tools that is used is so natural that they hardly even consider it as something extraordinary. Interviewees from the Green Party however show that same 'natural'-approach to offline methods. This may partly be dependent on the age of the members that were interviewed, and the age difference may well influence the parties' behaviour more generally. The average of a Green Party member is 48 years, compared to 38 years in the Pirate Party.

\subsubsection{Binding Online Decisions}

According to interviewees from the Green Party, online voting tools have so far been prevented by the expected costs. In the short term, holding assemblies is considered cheaper and easier to organise than to build, implement and maintain an online system. As a delegate explained:

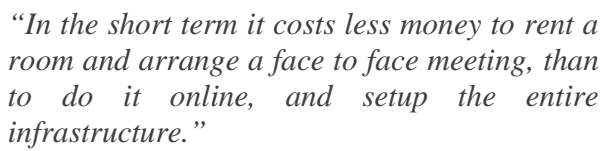

"In the short term it costs less money to rent a room and arrange a face to face meeting, than to do it online, and setup the entire infrastructure.”

In addition, all the arguments against online discussions are even more important for binding decisions. Nevertheless, according to a member of the party leadership, the party currently investigates if and how an online system for binding decisions could be implemented, and how exclusion through such a system could be prevented.

Despite many years of discussion, and the variety of tools that could potentially support such a step, the Pirate Party also cannot make legally binding online decisions. Liquid Feedback, although often described in the literature as a decision-making platform, does not have the statutory role that would be required to make binding decisions. It is used in the process, to develop policy and prepare motions, but not to vote on them. The one statutory option to make online decisions is the Basisentscheid (base decision). A member of the working group for the implementation of this solution described that, apart from internal legal complications, it has not been implemented due to conflicts within the party: software was developed for the purpose, but developers and executive board cannot agree on terms for its implementation.

\section{DISCUSSION}

In the following we will argue that in terms of democratic decisions

(1) both parties provide different opportunities for participation;

(2) participation in the Pirate Party is exclusive both online and offline;

(3) online and offline forms of participation can be mutually supportive;

(4) decisions in the Green Party are more likely to be accepted by the membership;

(5) the Green Party seems to follows collective action, the Pirate Party connective action principles;

(6) the lack of structure in the Pirate Party has led to unofficial leadership roles;

(7) gender differences in the Pirate Party persist systemically.

In regards to the use of technology,

(8) different participation profiles for different demographics are highly likely in the Pirate Party;

(9) online processes may have made participation easier in the Green Party, but not necessarily increased participation;

(10) an increase in participation by less technology-savvy members can be expected over time;

(11) the Pirate Party established online processes that fill equivalent roles to offline processes in the Green Party;

(12) The echo chamber effect online may lead to destabilisation;

(13) Liquid Democracy as a concept may be incompatible with democratic processes within political parties;

\subsection{Democratic Decisions}

(1) Neither of the parties uses a formal, purely deliberative decision-making process, but both of them use formal or informal deliberative elements. In the Green Party this is best visible in the consensus-meeting ahead of conferences, where motions are combined so that consensus can be achieved among delegates. The party offers opportunities for participation in their preparation of decisions, as every member can propose motions, but also through the delegate assemblies, where every member has a representative through which they are counted, even if absent [41]. In the Pirate Party, deliberation happens in a formal way only at general assemblies, through their structured discussion of motions, and is organised informally by members ahead of it.

The structure of the Green Party is very much geared towards democratic participation, with members being represented throughout decisions at all levels, apart from the local representative election, and additional direct input into the process in form of motion proposals and deliberation. The Pirate Party process on the other hand is geared towards direct participation, to the extent that members can directly participate at every stage of the process, but can do this only directly.

(2) Regardless of how participatory the online processes in the Pirate Party are, they are not inclusive, because they do not consider any differences in access or skills between members. The forum for actual binding decisions in form of offline general assemblies is equally exclusive, as it does not consider differences in mobility and funds between members. Comparable to what Smith and Gidden [36] found for assemblies in the Occupy movement, only those members who can attend the assembly, anywhere in the country, for an entire weekend, can speak and vote. Other than 
some social movements, the Pirate Party has not developed techniques such as the progressive stack [34], which could mitigate this situation by advancing the chances to be heard for the attending members of minorities.

(3) Different from Smith and Giddens findings for Occupy, developing motions and voting on them are probably not mutually exclusive, as general assemblies are planned far in advance and happen only once or twice per year. Decision preparation happens purely online, and consensus finding online is very much limited to Liquid Feedback - a tool that does not allow the exchange of arguments. The process engages the members that use the internet for motion proposition, and those who physically attend assemblies to vote upon them. Similar to what Gil de Zúñiga et al. [12] found for political bloggers, online and offline participation may be mutually supportive: The introduction and defence of motions at the assembly plays a vital role in the process. Members who propose motions, but cannot attend the assembly to introduce and defend them, may face a lower probability of their motions being accepted.

(4) In regards to Lunenburg's [27] advantages of group decision making, it can be argued that the decision-making process of the Green Party is likely to achieve a higher acceptance as well as more commitment from their members. The representation system may not lead to factual involvement of all members in decisions, but at least members can feel they are part of the decision through their representatives. While the Pirate Party involves members at every stage, this does not mean that every member is involved in every decision. Instead, members pick the working groups and motions they want to work on. When they do not attend the assembly, they do not actually participate in decisions, which may lead to frustration and reduced acceptance of decisions that are made in their absence.

(5) Considering the logic of collective and connective action by Bennett and Segerberg [3], it can be said that the parties, or rather their members, lean toward different principles. Both parties have a formal organisational structure, which is required of political parties by German law. Within that structure however, the Green Party has embraced institutions like representation and party wings, and although it encourages participation by individual members, the formal structure of branches and representatives takes priority. For the Pirate Party it is the other way around: although the formal organisation exists, the members refused further formalisation of structures such as party wings or representatives, and the interactions in the network of participating members is what carries the decision-making process.

(6) This is closely linked to the 'structurelessness' of the Pirate Party. The executive board does not have a special role in decisionmaking processes, and the guideline motions proposed by the Green Parties' board do not exist. ${ }^{1}$ Since there is no official leadership role in program development, unofficial leadership roles have emerged, as was expected following Freeman[38]. This behaviour is visible for example in the way members vote with their peers at the assembly. Despite clearly existing opinion leaders, these members do not have representative roles that would allow them to be held accountable or represent groups of members in deliberations between groups of opposing opinions.

(7) Regarding gender differences, the parties could hardly be more different. The Green Party focuses on equal participation for and representation of women in their policies as well as their actions, whereas the Pirate Party assumes that a uniform starting position for all members is sufficient to achieve equal participation for all members regardless of their gender. In combination with the lack of demographic data about their members, this is little surprising they neither know who their members are, nor who participates, and therefore cannot know how representative the participants are of the member base. The available data about the gender of members suggest that the vast majority of members are male, which in turn allows the assumption that the assumed equal participation may well be gendered assumptions [1]: The vast majority of male members defines and accepts the standards for participation as fair, therefore female members can be marginalised because they have no say in the setting of these standards, even if they disagree with them. Thus the problem is systemic and can hardly be addressed from within the existing processes.

\subsection{Use of Technology}

(8) The Green Party clearly acknowledges that online processes would exclude those members that do not use ICT, and that some members - especially those with staff at their disposal - derive greater benefits from online participation. Following Halford and Savage [16], knowledge about inequalities is required to mediate them. Since the Pirate Party does not consider these factors at all in their process design or decisions about technology they use, these inequalities are allowed to fester. Thus different participation profiles for different demographics, such as lower participation by single parents, older people, ethnic minorities or women, are highly likely.

(9) The fact that in the Green Party the number of motions did not change since the online process was introduced allows the assumption that the non-online members who wanted to propose motions have found ways to do this through other means. Thus the internet may have made the process easier for members who already participated, but did not necessarily lead to an increase in participation, in neither depth nor breadth.

(10) This strongly reminds of Kerr and Waddington [23]'s study of UNISON. Online participation in the Green Party might also start out with limited uptake in the beginning followed by a steady rise of participation by previously underrepresented groups. Then, a rise in participation of less online-proficient members in the Green Party can be expected.

(11) As predicted by Agre [2], the Green Party uses the web to amplify existing processes, some of which happens intentional, such as the introduction of the motion tool, and some is unintended, such as the use of Social Media to discuss motions. The Pirate Party on the other hand had no processes it could have amplified in the first place, and therefore developed entirely new processes, such as the refinement of motions through Liquid Feedback. It could be argued that the party found technological solutions for problems that may have arisen through their extended use of technology: They use online tools for nearly everything, which despite its opportunities, leads to a lack of face-to-face deliberation. These 'new processes' could then well be a product of necessity, created when gaps in the processes, such as consensus-development, needed to be addressed.

(12) Parties can be echo chambers, and as Weinberger [21] and Stevens and O’Hara [32] argue this need not be a negative effect. ${ }^{1}$ At the last assembly, the executive board of the Pirate Party did propose a motion comparable to the Green Parties' guideline motions,
which was accepted by the assembly. Since this happened after our data collection, we cannot include this new development in our analysis. 
Reinforcing the dominant views among members could have a stabilizing effect on internal political discourse. However, this can only work if the members truly have similar views. When the Green Party wings formed, the internet was not around yet. The failure of the Pirate Party to establish institutionalised wings, which could negotiate positions, may point to a new development. If online communication is indeed reinforcing dominant views, the echo chamber effect may also reinforce the differences between the views, thus decreasing the foundational agreement and destabilising the party. If this were the case, online communication would be a barrier to cooperation. If platforms themselves promote uniformity, controversial discussions may even be prevented by design, thus reducing the democracy in the process.

(13) Party wings could fill the role that Agre [2] saw for parties in democracy at large within parties. But where groups are not institutionalised and thus cannot be represented, consensus can neither be sought nor found. In their absence, the groups in the Pirate Party compete in size, and whichever group happens to be larger at assemblies forces their goals onto the other, and decisions are made by conflict rather than by consensus. If this is indeed the case, then the proposed advantages of Liquid Democracy, rather than leading to a more inclusive and participatory environment, may in fact result in fragmentation. Potentially, Liquid Democracy as a way of decision-making is not compatible with the organisational structure of a political party at all, since the very advantages of Liquid Democracy (more expertise, overcoming time and space limitations for face-to-face meetings, knowledge gaps of voters [28]), are contrary to one of the main advantages of political parties: to synthesise opinions into coherent platforms [2].

\section{CONCLUSION AND FUTURE WORK}

Processes required by law such as motion proposals by members, assemblies, or offline votes result in similarities among German parties. However, beyond that the two parties differ vastly, with very different effects on in- and exclusion. As we have shown, the internet does not by itself lead to more inclusion. To the contrary, new forms of exclusion can arise through differences in internet access, internet use, as well as the overwhelming amount of information made available through the web. Both cases we discussed are set in the specific context of the German political culture and political system. On the one hand, this context is very specific, so that conclusions beyond it should be drawn with care. On the other hand however, Germany is a Western democracy, and as such often the basis for democracy theory. In either case, the structure developed here may help to structure future work, which would need to see whether the differences found here are visible in a larger or differently composed sample.

The internet on its own does not determine the culture of a party, but it appears to have a strong influence on their process design. In turn, the culture of the party influences its adoption of technology. To a degree, party structure and internet technology can be mutually supportive, but this requires careful consideration of the existing party structure and culture. Both new processes and tools need to be designed in a way that is compatible with the current situation within the party. The best solution for online participation will always be dependent on specific use-cases. The goals, especially the perception of democratic decisions, need to be considered in process and tool design, in addition to the target group. Potential barriers and causes for exclusion should be discussed in early stages of the process.

Further work is needed to understand the effect of the internet on democratic decisions within parties better. For example, quantitative data could help to validate the satisfaction of members with the decisions made by assemblies, and the preferences and reasons for the acceptance of the different participation processes. Equally, the effects of specific internet technologies on in- and exclusion, such as the accessibility of the web over e.g. VoIP or other arbitrary layers, would warrant further investigation. A quantitative analysis of the overlap between the members that participate online, offline, and their demographic profile could give insight into the question whether different forms of participation can be predicted by demographics.

The biggest challenge remains to find a workable connection between both online and offline forms of participation, to prevent siloed discussions, benefit from the advantages of both forms, and stimulate more and broader participation overall.

\section{ACKNOWLEDGEMENTS}

We are grateful to our interview partners for their participation in this study. This research was funded by the EPSRC Centre for Doctoral Training in Web Science Innovation, EP/L016117/1. $\mathrm{KOH}$ and MLR were partly supported by EPSRC project SOCIAM, grant number EP/J017728/2.

\section{REFERENCES}

[1] Acker, J. 1990. HIERARCHIES, JOBS, BODIES: A Theory of Gendered Organizations. Gender \& Society. 4, 2 (Jun. 1990), 139-158.

[2] Agre, P.E. 2002. Real-Time Politics: The Internet and the Political Process. The Information Society. 18, 5 (2002), 311-331.

[3] Bennett, W.L. and Segerberg, A. 2012. THE LOGIC OF CONNECTIVE ACTION. Information, Communication \& Society. 15, 5 (Jun. 2012), 739-768.

Beschlussporotokoll AVB:

http://wiki.piratenpartei.de/BE:Parteita ssporotokoll_AVB\#Liste. Accessed: 2016-01-27.

[5] Buhl, H.U. 2011. From revolution to participation: Social media and the democratic decision-making process. Business and Information Systems Engineering. 3, 4 (2011), 195-198.

[6] Bullwinkel, B. and Probst, L. 2014. Innerparteiliche Willensbildung und Entscheidungsprozesse durch digitale Partizipation. Ein Praxistest des Konzepts der Liquid Democracy. Zeitschrift für Parlamentsfragen. 2, (2014), 382-401.

[7] Carr, L. et al. 2010. Could the Web be a Temporary Glitch? Proceedings of the WebSci10: Extending the Frontiers of Society On-Line, Raleigh, US, 26 - 27 Apr 2010 (Raleigh, US, 2010).

[8] Costanza-Chock, S. 2012. Mic Check! Media Cultures and the Occupy Movement. Social Movement Studies.

[9] Dahl, R. 1989. Democracy and its Critics. Yale University Press.

[10] Gibson, R. and Cantijoch, M. 2013. Conceptualizing and Measuring Participation in the Age of the Internet: Is Online Political Engagement Really Different to Offline? The Journal of Politics. 75, 03 (2013), 701-716.

[11] Gibson, Rachel K., Wainer Lusoli, Andrea Römmele, S.J.W. 2004. Introduction: representative democracy and the Internet. Electronic democracy: mobilisation, organisation and participation via new ICTs. R.K. Gibson et al., eds. Routledge / ECPR Studies in European Political Science. 1-16.

[12] Gil de Zúñiga, H. et al. 2010. Digital Democracy: 
Reimagining Pathways to Political Participation. Journal of Information Technology \& Politics. 7, 1 (2010), 36-51.

[13] Guthrie, K.K. and Dutton, W.H. 1992. The Politics of Citizen Access Technology. The Development of Public Information Utilities in Four Cities. Policy Studies Journal. 20, 4 (Dec. 1992), 574-597.

[14] Hague, B.N. and Loader, B.D. 1999. Digital Democracy: an introduction. Digital Democracy. Discourse and Decision Making in the Information Age. B.N. Hague and B.D. Loader, eds. Routledge. 3-22.

[15] Halford, S. et al. 2010. A manifesto for Web Science. Proceedings of the WebSci10: Extending the Frontiers of Society On-Line, Raleigh, US, 26 - 27 Apr 2010 (2010).

[16] Halford, S. and Savage, M. 2010. RECONCEPTUALIZING DIGITAL SOCIAL INEQUALITY. Information, Communication \& Society. 13, 7 (Oct. 2010), 937-955.

[17] Hardt, S. and Lopes, L.C.R. 2015. Google Votes : A Liquid Democracy Experiment on a Corporate Social Network. Technical Disclosure Commons.

[18] Hargittai, E. 2008. The Digital Reproduction of Inequality. Social Stratification. D. Grusky, ed. Westview Press. 936-944.

[19] Held, D. 2006. Models of Democracy. Stanford University Press.

[20] Hyland, J.L. 1995. Democratic Theory: The philosophical foundations. Manchester University Press.

[21] Is there an echo in here? 2004. http://www.salon.com/2004/02/21/echo_chamber/. Accessed: 2016-01-24.

[22] Jackson, N. and Lilleker, D. 2009. Building an Architecture of Participation? Political Parties and Web 2.0 in Britain. Journal of Information Technology Politics. 6, 3 (2009), 232-250.

[23] Kerr, A. and Waddington, J. 2014. E-Communications: An Aspect of Union Renewal or Merely Doing Things Electronically? British Journal of Industrial Relations. 52, 4 (Dec. 2014), 658-681.

[24] Kling, C.C. et al. 2015. Voting Behaviour and Power in Online Democracy: A Study of LiquidFeedback in Germany's Pirate Party. International Conference for Web and Social Media (ICWSM) in Oxford (2015).

[25] Kneuer, M. 2014. Mehr oder weniger demokratische Qualität durch das Internet? Der Bürger im Staat. 64, (2014).

[26] Lenk, K. 1999. Electronic support of citizen participation in planning processes. Digital Democracy. Discourse and Decision Making in the Information Age. B.N. Hague and B.D. Loader, eds. Routledge. 87-95.

[27] Lunenburg, F.C. 2011. Decision Making in Organizations. International journal of management, business, and administration. 15, 1 (2011), 1-9.

[28] Miller, J.C. 1969. A program for direct and proxy voting in the legislative process. Public Choice. 7, 1 (Sep. 1969), 107-113.
[29] Moore, R.K. 1999. Democracy and Cyberspace. Digital Democracy. Discourse and Decision Making in the Information Age. B.N. Hague and B.D. Loader, eds. Routledge. 39-59.

[30] Neumann, T. 2011. Die Piratenpartei Deutschland: Entwicklung und Selbstverständnis. Contumax.

[31] Niedermayer, O. 2015. Parteimitglieder in Deutschland:Version 2015. Arbeitshefte aus dem OttoStammer-Zentrum. 20, 25 (2015), 53.

[32] O’Hara, K. and Stevens, D. 2015. Echo Chambers and Online Radicalism: Assessing the Internet's Complicity in Violent Extremism. Policy Studies Organization. 9999, 9999 (2015), 1-22.

[33] Papacharissi, Z. 2009. The Virtual Sphere 2.0: The Internet, the Public Sphere and beyond. Routledge Handbook of Internet Politics. A. Chadwick and P.N. Howard, eds. Routledge. 230-245.

[34] Roth, S. et al. 2014. Occupy as a free space - Mobilization processes and outcomes. Sociological Research Online. 19, 1 (2014), 1-23.

[35] Schermerhorn, J.R.J. et al. 2011. Organizational Behavior. Wiley.

[36] Smith, J. and Glidden, B. 2012. Occupy Pittsburgh and the Challenges of Participatory Democracy. Social Movement Studies. 11, 3-4 (2012), 288-294.

[37] Spada, P. et al. 2014. A First Step toward Scaling-up Deliberation: Optimizing Large Group E-Deliberation using Argument Maps. Under Review. (2014), 36.

[38] The Tyranny of Structurelessness: http://www.jofreeman.com/joreen/tyranny.htm. Accessed: 2015-06-11.

[39] Thuermer 2015. Differences in decision-making processes between web-native and non-web-native political parties. University of Southampton.

[40] Ward, S. et al. 2002. Virtually participating: A survey of online party members. Information Polity: The International Journal of Government \& Democracy in the Information Age. 7, (2002), 199-215.

[41] Wilhelm, A.G. 1999. Virtual sounding boards: how deliberative is online political discussion? Digital Democracy. Discourse and Decision Making in the Information Age. B.N. Hague and B.D. Loader, eds. Routledge. 154-178.

[42] Wresch, W. 2001. Democracy in the Digital Age: Challenges to Political Life in Cyberspace. The Information Society. 17, 2 (2001), 145-146.

[43] Zhang, W. 2010. TECHNICAL CAPITAL AND PARTICIPATORY INEQUALITY IN EDELIBERATION. Information, Communication \& Society. 13, 7 (Oct. 2010), 1019-1039. 